Supporting Information for:

\title{
Routes to Acetonitrile-Supported Trifluoromethyl and Perfluorometallacyclopentane Complexes of Cobalt
}

Teng Xue and David A. Vicic*

Department of Chemistry, Lehigh University, 6 East Packer Ave, Bethlehem, Pennsylvania 18015, United States

Table of Contents

Pages S2-S6: NMR Spectral Data for All Compounds.

Pages S7-S9: Listing of $\mathrm{H} \cdots \mathrm{F}$ contacts shorter than the sum of van der Waals radii of $\mathrm{F}$ and $\mathrm{H}$ in all new structures. 
NMR Spectral Data for All Compounds:

Figure S1: ${ }^{19} \mathrm{~F}$ NMR of 5 in $\mathrm{CD}_{3} \mathrm{CN}$, collected on a spectrometer operating at $376 \mathrm{MHz}$, recorded at $25^{\circ} \mathrm{C}$, and referenced to $\alpha, \alpha, \alpha$-trifluorotoluene as an internal standard $(\delta=-63.7)$.

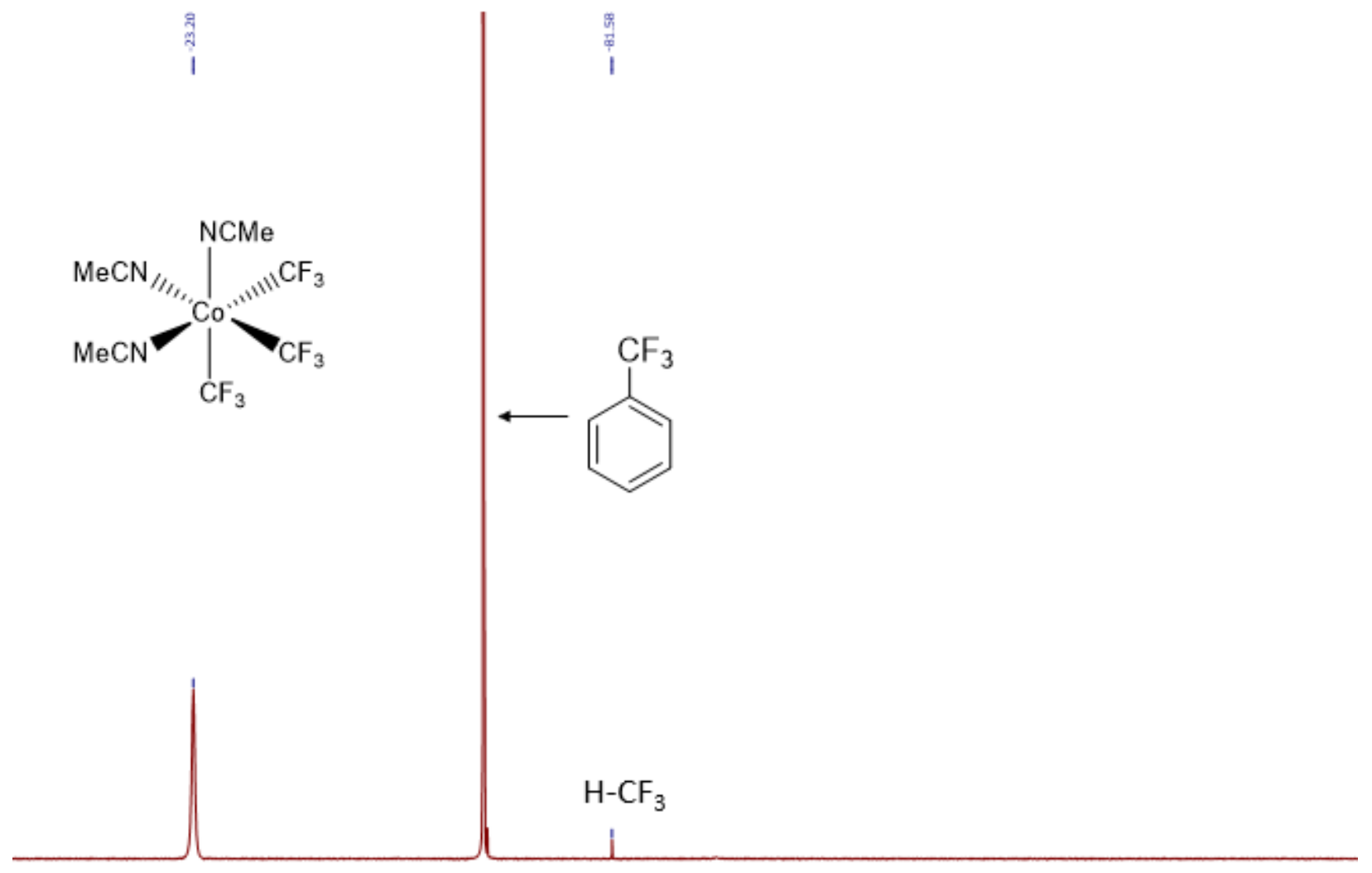

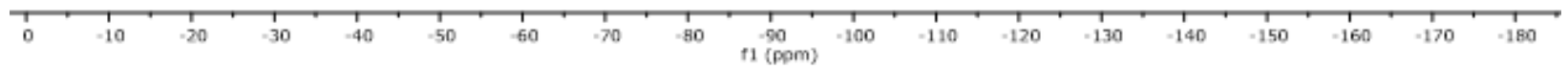


Figure S2: ${ }^{19} \mathrm{~F}$ NMR of $\mathbf{1 1}$ in $\mathrm{CD}_{3} \mathrm{CN}$ collected on a spectrometer operating at $376 \mathrm{MHz}$, recorded at $25^{\circ} \mathrm{C}$, and referenced to $\alpha, \alpha, \alpha$-trifluorotoluene as an internal standard $(\delta=-63.7)$.

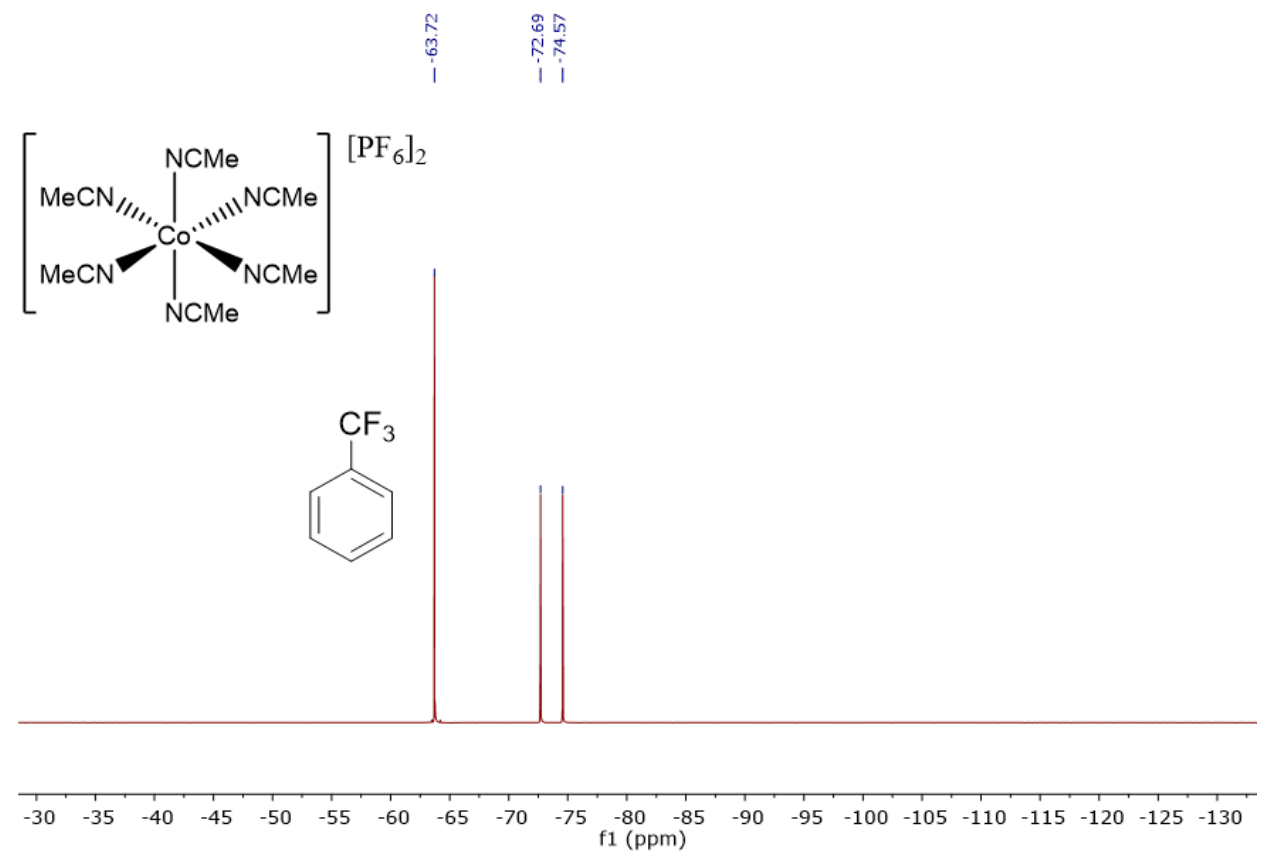


Figure S3: ${ }^{19} \mathrm{~F}$ NMR of 12 in $\mathrm{CD}_{3} \mathrm{CN}$, collected on a spectrometer operating at $376 \mathrm{MHz}$, recorded at $25^{\circ} \mathrm{C}$, and referenced to $\alpha, \alpha, \alpha$-trifluorotoluene as an internal standard $(\delta=-63.7)$.

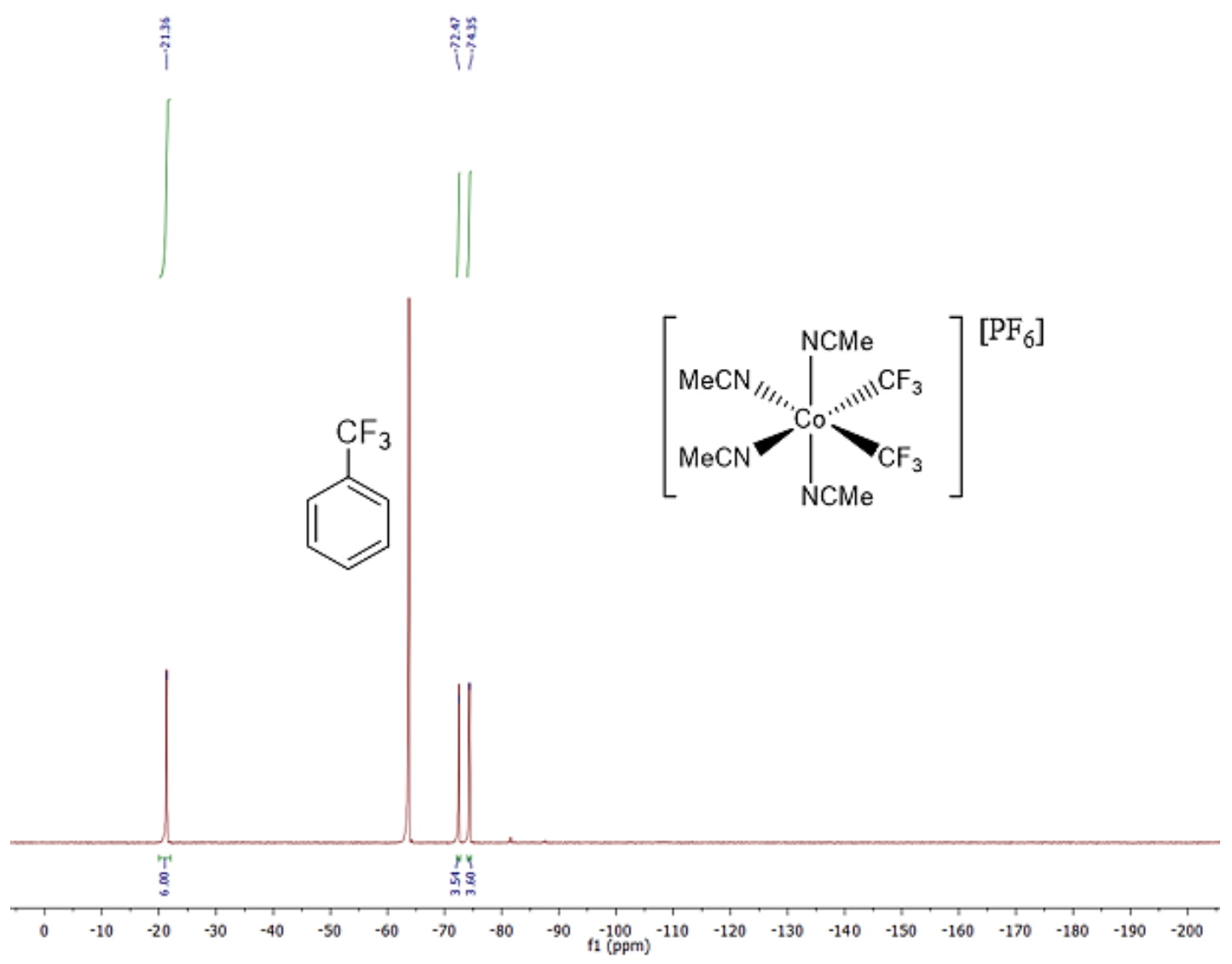


Figure S4: ${ }^{19} \mathrm{~F}$ NMR of 13-14 in $\mathrm{CD}_{3} \mathrm{CN}$, collected on a spectrometer operating at $376 \mathrm{MHz}$, recorded at $25^{\circ} \mathrm{C}$, and referenced to $\alpha, \alpha, \alpha$-trifluorotoluene as an internal standard $(\delta=-63.7)$.

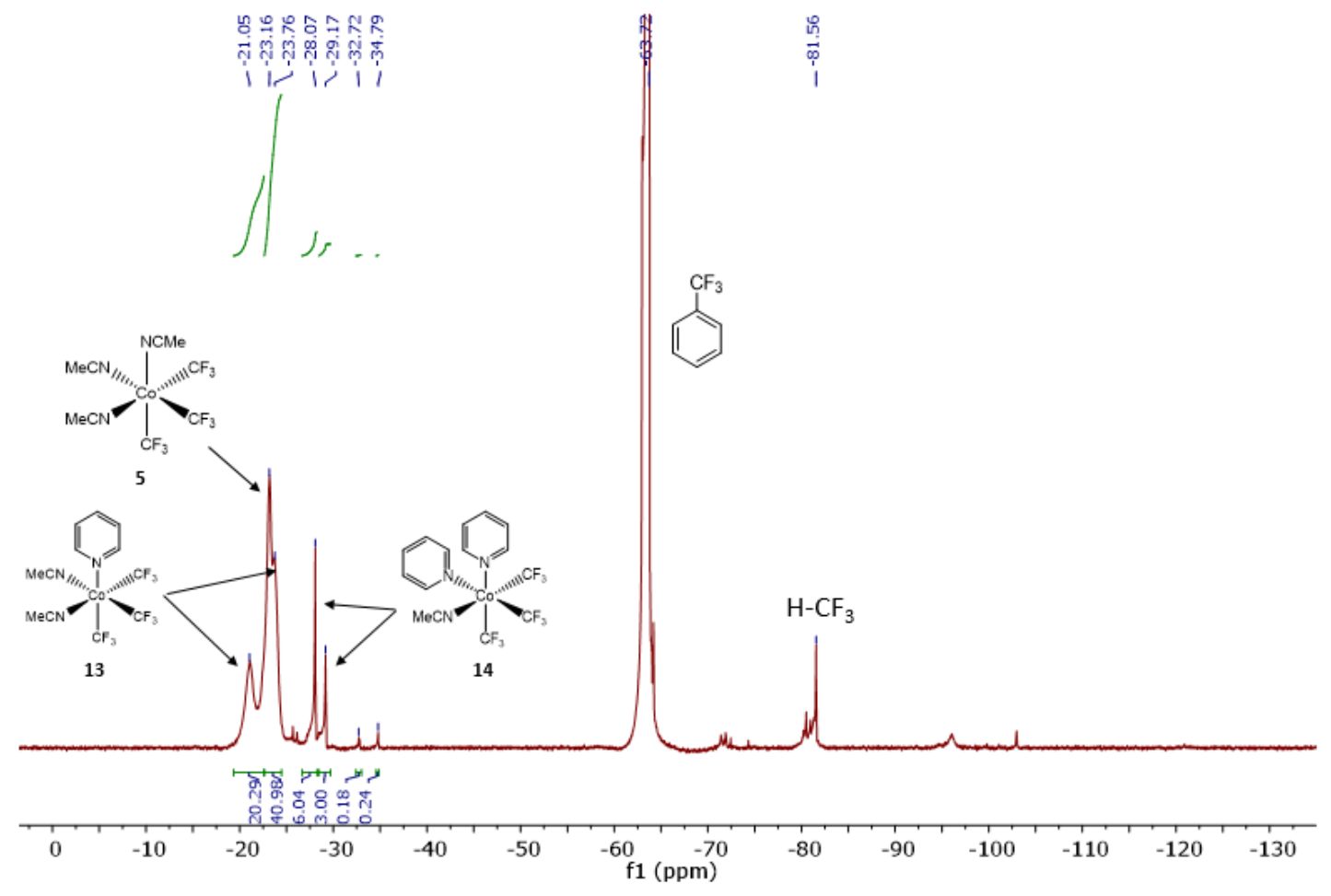


Figure S5: ${ }^{19} \mathrm{~F}$ NMR of 16 in $\mathrm{CD}_{3} \mathrm{CN}$, collected on a spectrometer operating at $376 \mathrm{MHz}$, recorded at $25^{\circ} \mathrm{C}$, and referenced to $\alpha, \alpha, \alpha$-trifluorotoluene as an internal standard $(\delta=-63.7)$.

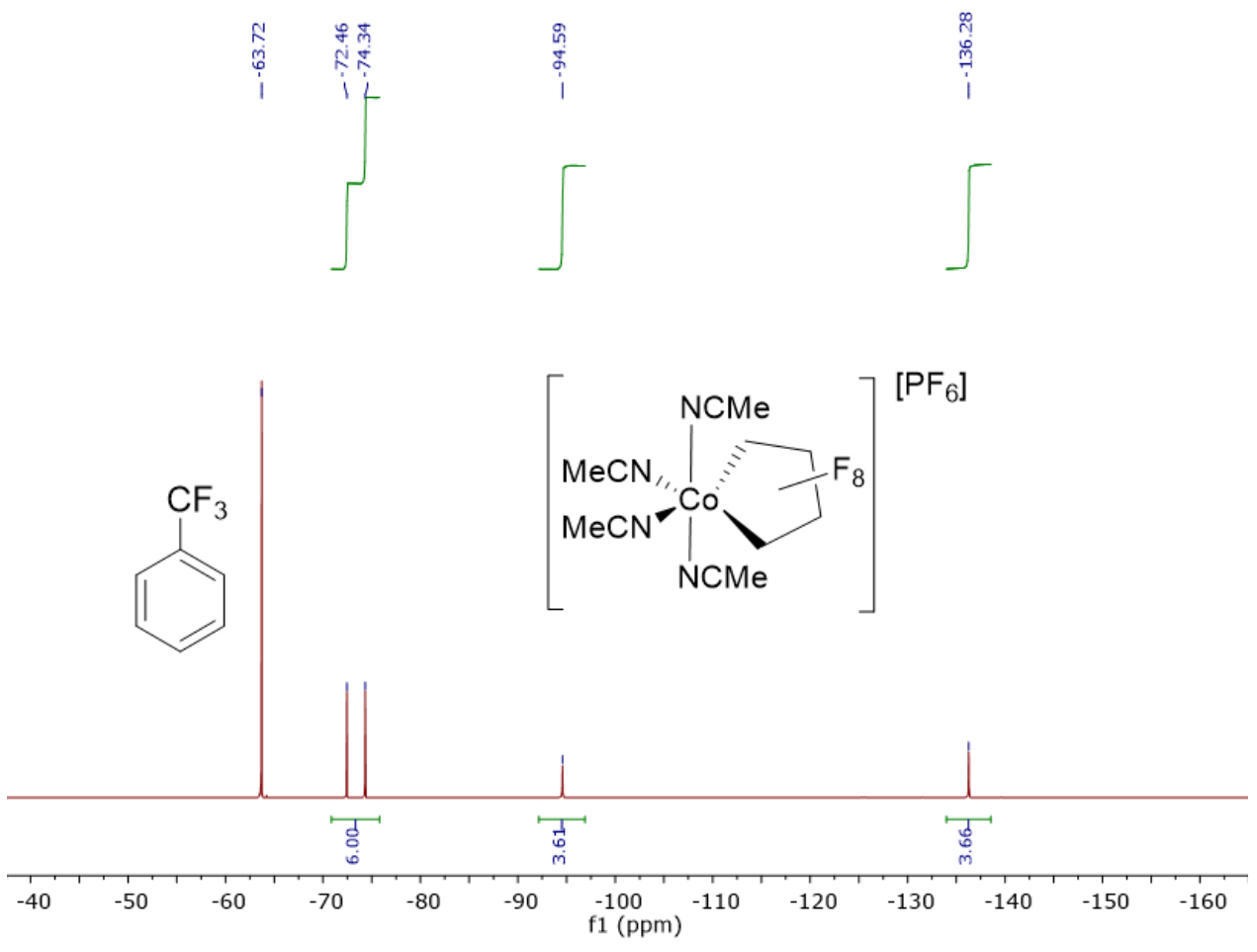


Table S1. Number of $\mathrm{H} \cdots \mathrm{F}$ contacts shorter than the sum of van der Waals radii of $\mathrm{F}$ and $\mathrm{H}$.

\begin{tabular}{|c|c|}
\hline Compound & \# of H...F contacts shorter than the sum of van der Waals radii of $\boldsymbol{F}$ and $\boldsymbol{H}$ \\
\hline $\mathbf{5}$ & 4 \\
\hline 12 & 8 \\
\hline 13 & 13 \\
\hline 14 & 8 \\
\hline 16 & 8 \\
\hline
\end{tabular}

Hydrogen contacts for Compound $\mathbf{5}$ with donor (D) and acceptor $(A)$ designations as determined by the SHELX program:

Hydrogen bonds with H..A $<r(A)+2.000$ Angstroms and $<\mathrm{DHA}>110 \mathrm{deg}$.

$\begin{array}{lccccl}\text { D-H } & d(D-H) & d(H . . A) & <D H A & d(D . . A) & A \\ \text { C5-H5B } & 0.980 & 2.505 & 136.45 & 3.285 & F 6[x-1 / 2,-y+3 / 2, z] \\ \text { C9-H9A } & 0.980 & 2.568 & 141.45 & 3.389 & F 6[-x+3 / 2, y+1 / 2, z-1 / 2] \\ \text { C9-H9B } & 0.980 & 2.613 & 151.37 & 3.504 & F 2[x-1 / 2,-y+3 / 2, z] \\ \text { C9-H9C } & 0.980 & 2.604 & 127.47 & 3.293 & F 7[-x+1,-y+1, z-1 / 2]\end{array}$

Hydrogen contacts for Compound 12 with donor (D) and acceptor (A) designations as determined by the SHELX program:

Hydrogen bonds with H..A $<r(A)+2.000$ Angstroms and $<D H A>110$ deg.

$\begin{array}{lccccl}\text { D-H } & d(D-H) & d(H . . A) & <D H A & d(D . . A) & A \\ \text { C4-H4A } & 0.980 & 2.373 & 150.40 & 3.261 & F 8[-x+1,-y+1,-z+1] \\ \text { C4-H4B } & 0.980 & 2.443 & 151.87 & 3.339 & F 9[x-1 / 2,-y+3 / 2,-z+1] \\ \text { C4-H4B } & 0.980 & 2.408 & 141.17 & 3.231 & F 11[x-1 / 2,-y+3 / 2,-z+1] \\ \text { C4-H4C } & 0.980 & 2.489 & 124.53 & 3.150 & F 8 \\ \text { C8-H6 } & 0.989 & 2.428 & 174.14 & 3.413 & F 6[-x+3 / 2, y-1 / 2, z] \\ \text { C10-H10A } & 0.980 & 2.581 & 133.09 & 3.328 & F 6[-x+1, y-1 / 2,-z+1 / 2] \\ \text { C10-H10B } & 0.980 & 2.587 & 135.94 & 3.361 & F 9[-x+1,-y+1,-z+1] \\ \text { C10-H10C } & 0.980 & 2.513 & 132.00 & 3.251 & F 10[-x+3 / 2, y-1 / 2, z]\end{array}$


Hydrogen contacts for Compound $\mathbf{1 3}$ with donor (D) and acceptor (A) designations as determined by the SHELX program:

Hydrogen bonds with H..A $<r(A)+2.000$ Angstroms and $<$ DHA $>110$ deg.

$\begin{array}{lllllll}\text { D-H } & d(D-H) & d(H . . A) & <D H A & d(D . . A) & A \\ \text { C5-H5 } & 0.950 & 2.632 & 121.32 & 3.230 & F 1[-x+1, y+1 / 2,-z+3 / 2] \\ \text { C7-H7 } & 0.950 & 2.637 & 111.17 & 3.109 & F 6[-x,-y+1,-z+1] \\ \text { C8-H8 } & 0.950 & 2.355 & 136.85 & 3.117 & F 6 \\ \text { C8-H8 } & 0.950 & 2.318 & 126.47 & 2.982 & F 6[-x,-y+1,-z+1] \\ \text { C10-H10A } & 0.980 & 2.628 & 159.08 & 3.561 & F 2[x+1, y, z] \\ \text { C10-H10A } & 0.980 & 2.589 & 114.85 & 3.130 & F 3[x+1, y, z] \\ \text { C10-H10B } & 0.980 & 2.356 & 143.78 & 3.200 & F 5[-x+1, y-1 / 2,-z+3 / 2] \\ \text { C10-H10B } & 0.980 & 2.520 & 136.49 & 3.300 & F 7[-x+1, y-1 / 2,-z+3 / 2] \\ \text { C10-H10C } & 0.980 & 2.602 & 137.08 & 3.386 & F 8[-x+1,-y+1,-z+2] \\ \text { C10-H10C } & 0.980 & 2.635 & 158.10 & 3.563 & F 9[-x+1,-y+1,-z+2] \\ \text { C12-H12B } & 0.980 & 2.563 & 164.81 & 3.518 & F 5[-x,-y+1,-z+1] \\ \text { C12-H12C } & 0.980 & 2.489 & 144.29 & 3.334 & F 4[-x, y-1 / 2,-z+3 / 2] \\ \text { C12-H12C } & 0.980 & 2.524 & 139.04 & 3.326 & F 9[-x, y-1 / 2,-z+3 / 2]\end{array}$

Hydrogen contacts for Compound $\mathbf{1 4}$ with donor (D) and acceptor (A) designations as determined by the SHELX program:

Hydrogen bonds with H..A $<r(A)+2.000$ Angstroms and $<$ DHA $>110$ deg.

$\begin{array}{lrrrrl}\text { D-H } & d(D-H) & d(H . . A) & <D H A & d(D . . A) & A \\ \text { C5-H5A } & 0.980 & 2.560 & 127.94 & 3.255 & F 1[x,-y+1 / 2, z-1 / 2] \\ \text { C5-H5A } & 0.980 & 2.596 & 122.75 & 3.233 & F 4[x,-y+1 / 2, z-1 / 2] \\ \text { C5-H5A } & 0.980 & 2.531 & 155.63 & 3.447 & F 7[x,-y+1 / 2, z-1 / 2] \\ \text { C5-H5B } & 0.980 & 2.406 & 166.74 & 3.367 & F 8[x, y-1, z] \\ \text { C6-H6 } & 0.950 & 2.477 & 125.64 & 3.127 & F 6\end{array}$




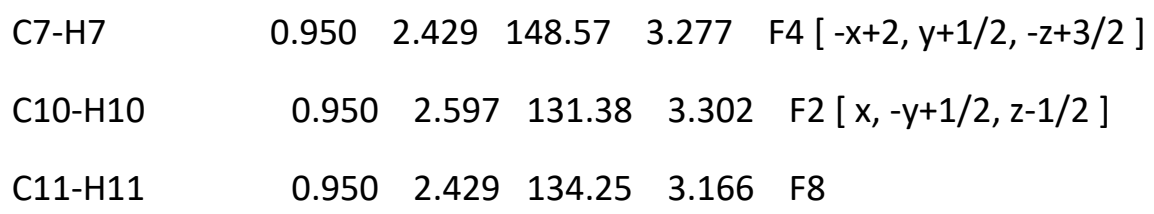

Hydrogen contacts for Compound $\mathbf{1 6}$ with donor (D) and acceptor (A) designations as determined by the SHELX program:

Hydrogen bonds with H..A $<r(A)+2.000$ Angstroms and $<\mathrm{DHA}>110 \mathrm{deg}$.

$\begin{array}{llllll}\text { D-H } & d(D-H) & d(H . . A) & <D H A & d(D . . A) & A \\ \text { C6-H6A } & 0.980 & 2.520 & 125.67 & 3.192 & F 1[-x+2,-y+1,-z+1] \\ \text { C6-H6C } & 0.980 & 2.599 & 129.69 & 3.312 & F 10[-x+1,-y+1,-z] \\ \text { C8-H8A } & 0.980 & 2.518 & 170.72 & 3.489 & F 12[-x+1,-y+1,-z+1] \\ \text { C8-H8B } & 0.980 & 2.553 & 143.55 & 3.391 & F 13 \\ \text { C8-H8C } & 0.980 & 2.469 & 158.91 & 3.402 & F 8[x,-y+3 / 2, z+1 / 2] \\ \text { C10-H10B } & 0.980 & 2.612 & 113.72 & 3.137 & F 7[-x+1,-y+1,-z] \\ \text { C12-H12B } & 0.980 & 2.427 & 142.56 & 3.260 & F 9[-x+1,-y+1,-z+1] \\ \text { C12-H12C } & 0.980 & 2.639 & 123.95 & 3.289 & F 5[-x+2,-y+1,-z+1]\end{array}$

